\title{
STATE SPACE VECTOR BASED ADVANCED DIRECT POWER CONTROL OF MATRIX CONVERTER AS UPFC
}

\author{
P. Lakshmi Bhargavi ${ }^{1,}$ S. Suresh Reddy ${ }^{2,}$ S. Sarat Kumar Sahu ${ }^{3}$ \\ ${ }^{1}$ PGScholar,Dept of PS (EEE), ${ }^{2}$ Head of the Dept of EEE, ${ }^{3}$ Professor,Dept of EEE, NBKR, Vidyanagar, APMVGR, \\ Vizayanagaram, AP, padarthi224@gmail.com, sanna_suresh@reddifmail.com,sahu.sarat@gmail.com
}

\begin{abstract}
This paper presents state space vector analysis for three-phase matrix converters operating as unified power flow controllers (UPFCs). It allows direct ac/ac power conversion without dc energy storage links; therefore, It reduces volume, cost, capacitor power losses, together with higher reliability. The line active and reactive power, together with ac supply reactive power, can be directly controlled by selecting an appropriate matrix converter switching state guaranteeing good steady-state and dynamic responses. This advanced control of MC guarantee faster responses without overshoot and no steady- state error, presenting no cross-coupling in dynamic and steady-state responses. Simulations are carried out, showing the effectiveness of the proposed method in steady-state and transient conditions.
\end{abstract}

Keywords: Direct power control (DPC), matrix converter (MC), unified power flow controller (UPFC), state space vectors.

-***

\section{INTRODUCTION}

In the last few years Flexible AC Transmission Systems (FACTS) became well known powerelectronicsbasedequipmenttocontroltransmissionlinespowerflow. UPFCs are the most versatile and complex FACTS allowing precise and reliable control of both active and reactive power flow over the network .UPFC can prevail over line impedance dependencies, sending and receiving end voltage amplitudes and phase differences. The original UPFC concept, introduced in the nineties by L. Gyugyi[1], consists of two ACDCconverters using Gate- Turn Off thyristors(GTO), back to back connected through their common DC link using large high-voltage DC storage capacitors. Both converters AC sides are connected to the transmission line, through coupling transformers, in shunt and series connection with the line.

This arrangement can be operated as an ideal reversible ACAC switching power converter, in which the power can flow in either direction between the AC terminals of the two converters. The DC link capacitors provide some energy storage capability to the back to back converters that help the power flow control Replacing the two three-phase inverters by one matrix converter the DC link(bulk) capacitors are eliminated,reducing,costs,size, maintenance, increasingreliabili tyandlifetime.TheAC-ACmatrixconverter, also known as all silicon converters , processes the energy directly without large energy storage needs. This leads to an increase of the matrix converter control complexity. In [2] an UPFC-connected power transmission network model was proposed with matrix converters and in[3] was used to synthesize both active $(\mathrm{P})$ and reactive $(\mathrm{Q})$ power controllers using a modified Venturini high-frequency PWM modulator. In this paper a Matrix Converter based UPFC-connected power transmission network model is proposed, using a Direct Power Control approach(DPC-MC). This control method is based on sliding mode control techniques [5] and allows real time selection of adequate state-space vectors to control input and output variables. Transmission line active and reactive power flow can be directly controlled using this approach and the dynamic and steady state behaviour of the proposed P,Q control method is evaluated and discussed using detailed simulations. Results shows decoupled active and reactive power control, zero error tracking and fast response times.

\section{MODELLING OF THE UPFC POWER SYSTEM}

\section{General Architecture}

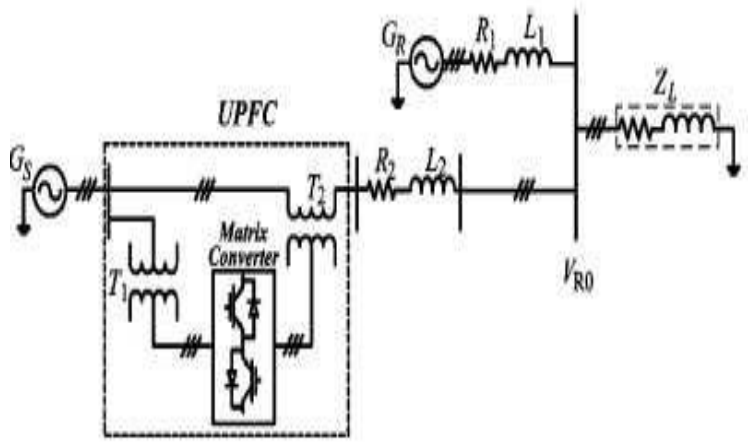

Fig.1.Transmission network with matrix converter UPFC 


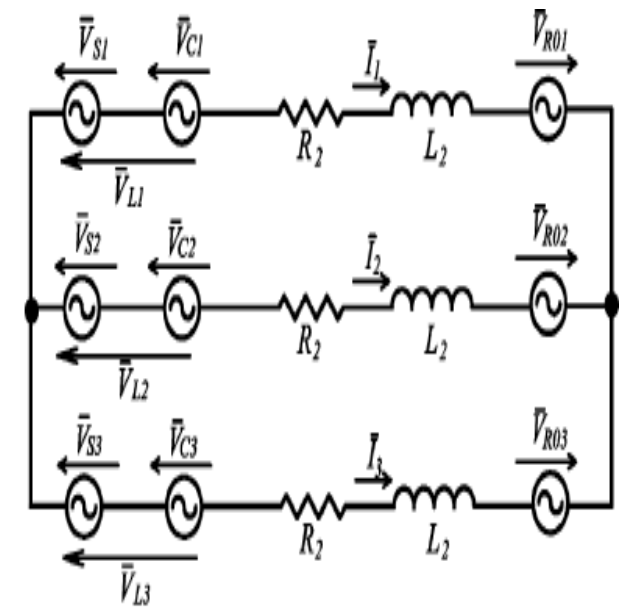

Fig.2.Three phase equivalent circuit of the matrix UPFC and transmission line

matrix converter is considered ideal and represented as a controllable voltage source, with amplitude Vc and phase $\rho$. In the equivalent circuit, VRois the load bus voltage. The DPCMC controller will treat the simplified elements as disturbances.

Considering a symmetrical and balanced three-phase system and applying Kirchhoff laws (Fig.2.), the ac line currents are obtained in dq coordinates as (1) and (2) equations.

$$
\begin{aligned}
& \frac{d I_{d}}{d t}=\omega I_{q}-\frac{R_{2}}{L_{2}} I_{d}+\frac{1}{L_{2}}\left(V_{L d}-V_{R o d}\right) \ldots \ldots . \\
& \frac{d I_{q}}{d t}=-\omega I_{d}-\frac{R_{2}}{L_{2}} I_{q}+\frac{1}{L_{2}}\left(V_{L q}-V_{R o q}\right)
\end{aligned}
$$

The active and reactive power of sending end generator are given in dq coordinates

$$
\left[\begin{array}{l}
P \\
Q
\end{array}\right]=\left[\begin{array}{cc}
V_{d} & V_{q} \\
V_{q} & -V_{d}
\end{array}\right]\left[\begin{array}{l}
I_{d} \\
I_{q}
\end{array}\right]
$$

Assuming $V_{R o d}$ and $V_{S d}=V_{d}$ as constants and a rotating reference frame synchronised to the $V_{s}$ source so that $V_{S q}=0$, active and reactive power $\mathrm{P}$ and $\mathrm{Q}$ are given by (4) and (5),respectively.

$$
\begin{aligned}
& P=V_{d} I_{d} \\
& Q=-V_{d} I_{q}
\end{aligned}
$$

Based on the desired active and reactive power (Pref, Qref), reference current (Idref, Iqref) can be calculated from (4)and(5)for current controllers. However, allowing P,Q actual powers are sensitive to errors in the $\mathrm{Vd}, \mathrm{Vq}$ values.

\section{B. Matrix converter output voltage and input current vectors}

(Fig.3) includes the three-phase shunt input transformer(with windings $\mathrm{Ta}, \mathrm{Tb}, \mathrm{Tc})$, the three-phase series output transformer(with windings TA,TB,TC),and the three-phase matrix converter, represented as an array of nine bidirectional switches Skj with turn-on and turn-off capability, allowing the connection of each one of three output phases directly to any one of the three input phases. The three-phase (ICr)input filter is required to re-establish a voltage-source boundary to the matrix converter, enabling smooth input currents.

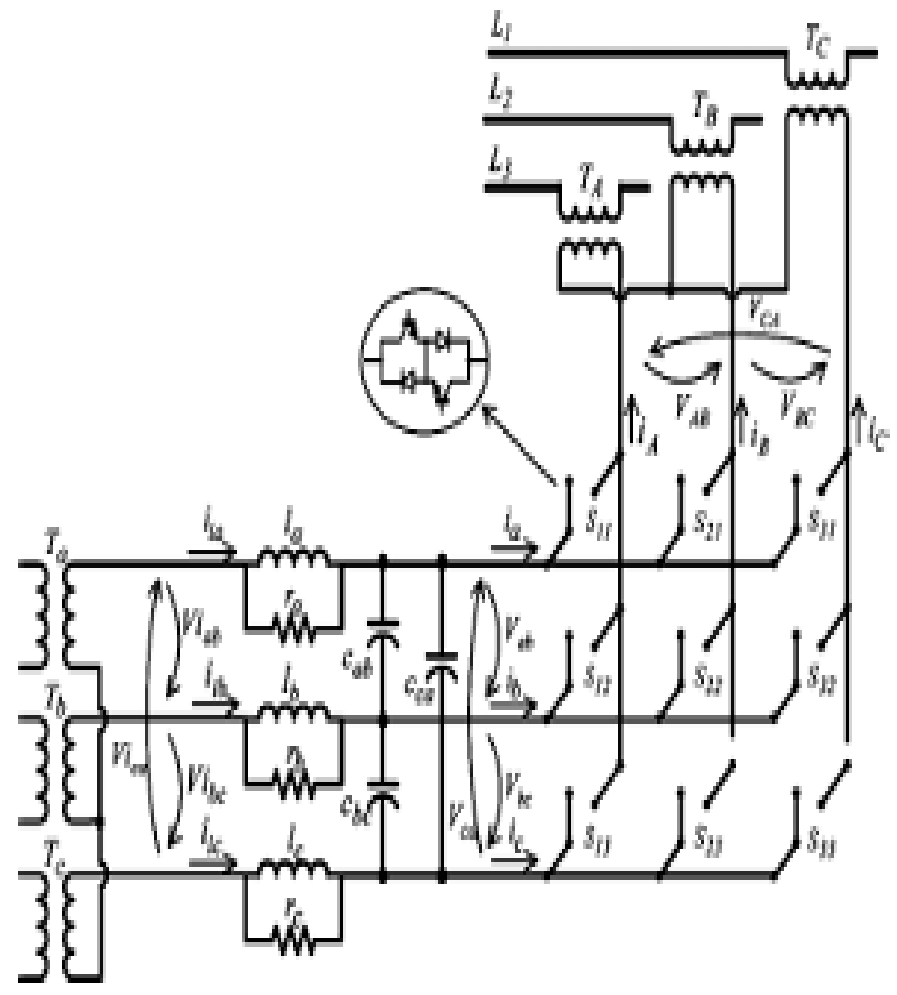

Fig3.Transmission network with matrix converter UPFC

Applying dq coordinates to the input filter state variables and neglecting the effects of the damping resistors the equations obtained are:

$$
\frac{d i_{i d}}{d t}=\omega_{i q}-\frac{1}{2 l} V_{d}-\frac{1}{2 \sqrt{3} l} V_{q}+\frac{1}{l} V_{i d}
$$




$$
\begin{gathered}
\frac{d i_{i q}}{d t}=-\omega i_{i d}-\frac{1}{2 l} V_{q}+\frac{1}{2 \sqrt{3} l} V_{d}+\frac{1}{l} V_{i q} \\
\frac{d V_{d}}{d t}=\omega V_{q}+\frac{1}{2 C} i_{i d}-\frac{1}{2 \sqrt{3} C} i_{i q}+\frac{1}{2 \sqrt{3} C} i_{q} \\
\frac{d V_{q}}{d t}=-\omega V_{d}+\frac{1}{2 C} i_{i q}+\frac{1}{2 \sqrt{3} C} i_{i d} \\
\frac{1}{2 \sqrt{3} C} i_{d}-\frac{1}{2 C} i_{q}
\end{gathered}
$$

Where $V_{\text {id }}, V_{\text {iq }}, i_{i d}, i_{i q}$ represents input Voltages and currents in dq components $\mathrm{Vd}, \mathrm{Vq}$, id, iq the matrix converter voltages and input current in dq components, respectively.

Assuming ideal semi conductors, each matrix converter bi directional switch $\mathrm{Skj}$ can assume two possible states:"Skj=1"iftheswitchisclosed or "Skj=0" if the switch is open. The nine matrix converter switches can be represented as a $3 * 3$ matrix $(7)$

$$
\boldsymbol{S}=\left[\begin{array}{c}
S_{11} S_{12} S_{13} \\
S_{21} S_{22} S_{23} \\
S_{31} S_{32} S_{33}
\end{array}\right](7)
$$

The relationship between load and input voltages can be expressed as:

$$
\left[\mathrm{v}_{\mathrm{A}} \mathrm{v}_{\mathrm{B}} \mathrm{v}_{\mathrm{C}}\right] \mathrm{T}=\mathrm{S}\left[\mathrm{v}_{\mathrm{a}} \mathrm{v}_{\mathrm{b}} \mathrm{v}_{\mathrm{c}}\right] \mathrm{T}
$$

The input phase currents can be related to the output phase currents (9), using the transpose of matrix S

$$
\left[\mathrm{i}_{\mathrm{a}} \mathrm{i}_{\mathrm{b}} \mathrm{i}_{\mathrm{c}}\right] \mathrm{T}=\mathrm{ST}\left[\mathrm{i}_{\mathrm{A}} \mathrm{i}_{\mathrm{B}} \mathrm{i}_{\mathrm{C}}\right] \mathrm{T}
$$

From the 27 possible switching patterns, time-variant vectors can be obtained (Table:I) representing the matrix output voltages and input currents in $\alpha \beta$ frame [fig 4(b)].

The active and reactive power DPC-MC will select one of these 27 vectors at any given time instant. 


\section{Switching Combinations and output voltage/Input Current state-Space Vectors （Table I)}

\begin{tabular}{|c|c|c|c|c|c|c|c|c|c|c|c|c|c|c|}
\hline Group & Name & $A$ & $B$ & $C$ & $v_{A B}$ & $v_{B C}$ & $v_{C A}$ & $i_{a}$ & $i_{b}$ & $i_{c}$ & $V_{o}$ & $\delta_{o}$ & $I_{i}$ & $\mu_{i}$ \\
\hline \multirow{6}{*}{ I } & $1 \mathrm{~g}$ & $\mathrm{a}$ & $b$ & $\mathrm{c}$ & $v_{a b}$ & $v_{b c}$ & $v_{c a}$ & $i_{A}$ & $i_{B}$ & $i_{C}$ & $v_{i}$ & $\delta_{i}$ & $\sqrt{3} i_{o}$ & $\mu_{o}$ \\
\hline & $2 \mathrm{~g}$ & a & $\mathrm{c}$ & b & $-v_{c a}$ & $-v_{b c}$ & $-v_{a b}$ & $i_{A}$ & $i_{C}$ & $i_{B}$ & $-v_{i}$ & $-\delta_{i}+4 \pi / 3$ & $\sqrt{3} i_{o}$ & $-\mu_{o}$ \\
\hline & $3 \mathrm{~g}$ & b & a & c & $-v_{a b}$ & $-v_{c a}$ & $-v_{b c}$ & $i_{B}$ & $i_{A}$ & $i_{C}$ & $-v_{i}$ & $\delta_{i}$ & $\sqrt{3} i_{0}$ & $-\mu_{o}+2 \pi / 3$ \\
\hline & $4 \mathrm{~g}$ & b & $\mathrm{c}$ & a & $v_{b c}$ & $v_{c a}$ & $v_{a b}$ & $i_{C}$ & $i_{A}$ & $i_{B}$ & $v_{i}$ & $\delta_{i}+4 \pi / 3$ & $\sqrt{3} i_{o}$ & $\mu_{o}+2 \pi / 3$ \\
\hline & $5 \mathrm{~g}$ & c & a & b & $v_{c a}$ & $v_{a b}$ & $v_{b c}$ & $i_{B}$ & $i_{C}$ & $i_{A}$ & $v_{i}$ & $\delta_{i}+2 \pi / 3$ & $\sqrt{3} i_{o}$ & $\mu_{o}+4 \pi / 3$ \\
\hline & $6 \mathrm{~g}$ & c & $\mathrm{b}$ & a & $-v_{b c}$ & $-v_{a b}$ & $-v_{c a t}$ & $i_{C}$ & $i_{B}$ & $i_{A}$ & $-v_{i}$ & $-\delta_{i}+2 \pi / 3$ & $\sqrt{3} i_{o}$ & $-\mu_{o}+4 \pi / 3$ \\
\hline \multirow{18}{*}{ П } & +1 & a & $\mathrm{b}$ & $\mathrm{b}$ & $v_{a b}$ & 0 & $-v_{a b}$ & $i_{A}$ & $-i_{A}$ & 0 & $\sqrt{2 / 3} v_{a b}$ & 0 & $\sqrt{2} i_{A}$ & $-\pi / 6$ \\
\hline & -1 & b & a & a & $-v_{a b}$ & 0 & $v_{a b}$ & $-i_{A}$ & $i_{A}$ & 0 & $-\sqrt{2 / 3} v_{a b}$ & 0 & $-\sqrt{2} i_{A}$ & $-\pi / 6$ \\
\hline & +2 & b & $\mathrm{c}$ & $\mathrm{c}$ & $v_{b c}$ & 0 & $-v_{b c}$ & 0 & $i_{A}$ & $-i_{A}$ & $\sqrt{2 / 3} v_{b c}$ & 0 & $\sqrt{2} i_{A}$ & $\pi / 2$ \\
\hline & -2 & c & $\mathrm{b}$ & b & $-v_{b c}$ & 0 & $v_{b c}$ & 0 & $-i_{A}$ & $i_{A}$ & $-\sqrt{2 / 3} v_{b c}$ & 0 & $-\sqrt{2} i_{A}$ & $\pi / 2$ \\
\hline & +3 & c & a & a & $v_{c a}$ & 0 & $-v_{c a}$ & $-i_{A}$ & 0 & $i_{A}$ & $\sqrt{2 / 3} v_{c a}$ & 0 & $\sqrt{2} i_{A}$ & $7 \pi / 6$ \\
\hline & -3 & a & $\mathrm{c}$ & $\mathrm{c}$ & $-v_{c a}$ & 0 & $v_{c a}$ & $i_{A}$ & 0 & $-i_{A}$ & $-\sqrt{2 / 3} v_{c a}$ & 0 & $-\sqrt{2} i_{A}$ & $7 \pi / 6$ \\
\hline & +4 & b & a & b & $-v_{a b}$ & $v_{a b}$ & 0 & $i_{B}$ & $-i_{B}$ & 0 & $\sqrt{2 / 3} v_{a b}$ & $2 \pi / 3$ & $\sqrt{2} i_{B}$ & $-\pi / 6$ \\
\hline & -4 & a & $\mathrm{b}$ & a & $v_{a b}$ & $-v_{a b}$ & 0 & $-i_{B}$ & $i_{B}$ & 0 & $-\sqrt{2 / 3} v_{a b}$ & $2 \pi / 3$ & $-\sqrt{2} i_{B}$ & $-\pi / 6$ \\
\hline & +5 & $\mathrm{c}$ & $\mathrm{b}$ & c & $-v_{b c}$ & $v_{b c}$ & 0 & 0 & $\dot{i}_{B}$ & $-i_{B}$ & $\sqrt{2 / 3} v_{b c}$ & $2 \pi / 3$ & $\sqrt{2} i_{B}$ & $\pi / 2$ \\
\hline & -5 & $\mathrm{~b}$ & $\mathrm{c}$ & b & $v_{b c}$ & $-v_{b c}$ & 0 & 0 & $-i_{B}$ & $i_{B}$ & $-\sqrt{2 / 3} v_{b c}$ & $2 \pi / 3$ & $-\sqrt{2} i_{B}$ & $\pi / 2$ \\
\hline & +6 & $\mathrm{a}$ & $\mathrm{c}$ & a & $-v_{c a}$ & $v_{c a}$ & 0 & $-i_{B}$ & 0 & $i_{B}$ & $\sqrt{2 / 3} v_{c a}$ & $2 \pi / 3$ & $\sqrt{2} i_{B}$ & $7 \pi / 6$ \\
\hline & -6 & c & a & $\mathrm{c}$ & $v_{c a}$ & $-v_{c a}$ & 0 & $i_{B}$ & 0 & $-i_{B}$ & $-\sqrt{2 / 3} v_{c a}$ & $2 \pi / 3$ & $-\sqrt{2} i_{B}$ & $7 \pi / 6$ \\
\hline & +7 & $\mathrm{~b}$ & $\mathrm{~b}$ & a & 0 & $-v_{a b}$ & $v_{a b}$ & $i_{C}$ & $-i_{C}$ & 0 & $\sqrt{2 / 3} v_{a b}$ & $4 \pi / 3$ & $\sqrt{2} i_{C}$ & $-\pi / 6$ \\
\hline & -7 & a & a & b & 0 & $v_{a b}$ & $-v_{a b}$ & $-i_{C}$ & $i_{C}$ & 0 & $-\sqrt{2 / 3} v_{a b}$ & $4 \pi / 3$ & $-\sqrt{2} i_{C}$ & $-\pi / 6$ \\
\hline & +8 & $\mathrm{c}$ & $\mathrm{c}$ & $\mathrm{b}$ & 0 & $-v_{b c}$ & $v_{b c}$ & 0 & $i_{C}$ & $-i_{C}$ & $\sqrt{2 / 3} v_{b c}$ & $4 \pi / 3$ & $\sqrt{2} i_{C}$ & $\pi / 2$ \\
\hline & -8 & b & $\mathrm{b}$ & $\mathrm{c}$ & 0 & $v_{b c}$ & $-v_{b c}$ & 0 & $-i_{C}$ & $i_{C}$ & $-\sqrt{2 / 3} v_{b c}$ & $4 \pi / 3$ & $-\sqrt{2} i_{C}$ & $\pi / 2$ \\
\hline & +9 & a & a & $\mathrm{c}$ & 0 & $-v_{c a}$ & $v_{c a}$ & $-i_{C}$ & 0 & $i_{C}$ & $\sqrt{2 / 3} v_{c a}$ & $4 \pi / 3$ & $\sqrt{2} i_{C}$ & $7 \pi / 6$ \\
\hline & -9 & $\mathrm{c}$ & $\mathrm{c}$ & a & 0 & $v_{c a}$ & $-v_{c a}$ & $i_{C}$ & 0 & $-i_{C}$ & $-\sqrt{2 / 3} v_{c a}$ & $4 \pi / 3$ & $-\sqrt{2} i_{C}$ & $7 \pi / 6$ \\
\hline \multirow{3}{*}{ III } & $\mathrm{z}_{\mathrm{a}}$ & a & $\mathrm{a}$ & $\mathrm{a}$ & 0 & 0 & 0 & 0 & 0 & 0 & 0 & - & 0 & - \\
\hline & $z_{b}$ & b & $\mathrm{b}$ & b & 0 & 0 & 0 & 0 & 0 & 0 & 0 & - & 0 & - \\
\hline & $z_{c}$ & c & $\mathrm{c}$ & $\mathrm{c}$ & 0 & 0 & 0 & 0 & 0 & 0 & 0 & - & 0 & - \\
\hline
\end{tabular}

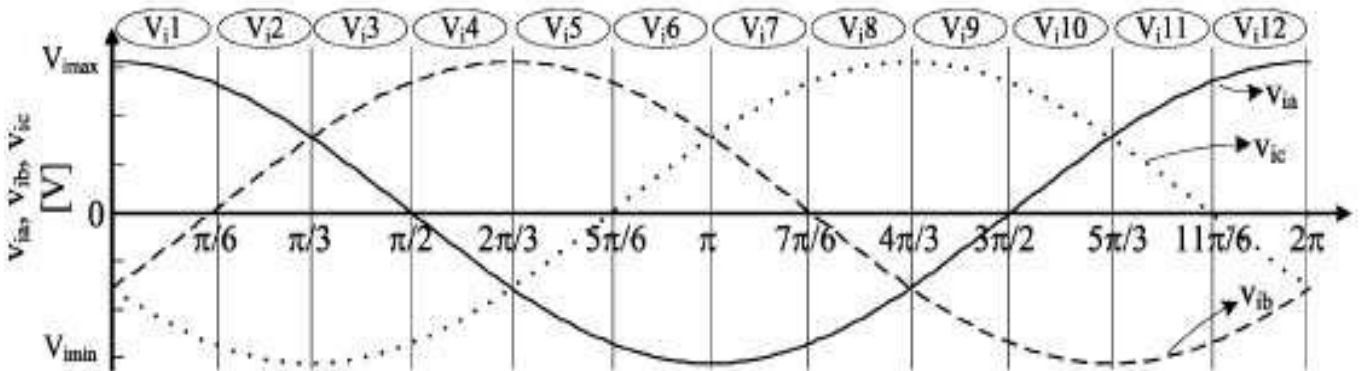

a)

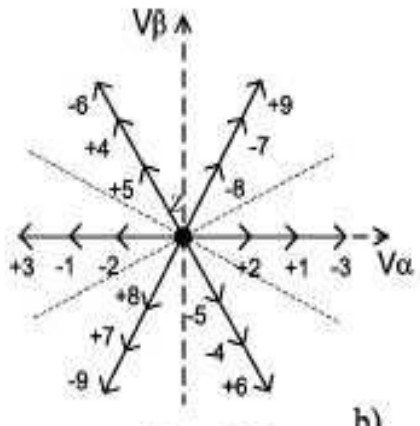

Sector $\mathbf{V}_{1} \mathbf{1}$ b)

Fig4: (a)Input voltages and their corresponding sector.(b)Output voltage state Space vectors when the input voltages a relocated at sector $\mathrm{V}_{\mathrm{i} 1}$ 


\section{SPACE VECTORCONTROL OF MC-UPFC}

\section{A. Line Active and Reactive Power Sliding Surfaces}

From Fig.2, in steady state, $V_{\text {dis }}$ imposed by source Vs. From (1)and (2),the transmission-line current can be considered as state variables with first-order dynamics dependent on the sources and time constant of impedance L2/R2 . Therefore, transmission-line active and reactive powers present firstorder dynamics and have a strong relative degree of one, since from the control view point, its first time derivative already contains the control variable from the sliding mode control theory, robust sliding surfaces to control the $\mathrm{P}$ and $\mathrm{Q}$ variables with a relatively strong degree of one can be obtain, considering proportionality to a linear combination of the errors of the state variables.

Therefore define the active power error $e_{P}$ and the reactive power $e_{Q}$ as the difference between the power references Pref, Qref and the actual transmitted powers $P, Q$ respectively

$$
\begin{gathered}
e_{P}=\text { Pref }-\mathrm{P}(10) \\
e_{Q}=\text { Qref }-\mathrm{Q}
\end{gathered}
$$

The robust sliding surfaces $\operatorname{SP}\left(e_{P, \mathrm{t}}\right)$, and $\operatorname{SQ}\left(e_{Q}, \mathrm{t}\right)$, must be proportional to these errors, being zero after reaching sliding mode

$$
\begin{gathered}
\mathrm{SP}\left(e_{P, \mathrm{t}}\right)=k_{\mathrm{P}}\left(\mathrm{P}_{\text {ref }}-\mathrm{P}\right)=0 \\
\mathrm{SQ}\left(e_{Q, \mathrm{t})}=k_{\mathrm{Q}}(\mathrm{Q} \text { ref }-\mathrm{Q})=0\right.
\end{gathered}
$$

The proportionality gains $k_{\mathrm{P}}$ and $k_{\mathrm{Q}}$ are chosen to impose appropriate switching frequencies.

\section{B. Line Active and Reactive Power Direct Switching}

\section{Laws}

Based on errors eP and eQ to select in real time the matrix converter switching states.

To guarantee stability for active power and reactive power controllers the sliding mode stability conditions (14) and (15) must be verified.

$$
\begin{aligned}
& S P(e P, t) \dot{S P}(e P, t)<0 \\
& S Q(e Q, t) \dot{S Q}(e Q, t)<0
\end{aligned}
$$

a) To choose a vector from (4) and (12), and considering Pref and $\mathrm{Vd}$ in steady state, the following can be written:

$$
\dot{S P}(e P, t)=k P\left(\frac{d P r e f}{d t}-\frac{d P}{d t}\right)=-k P \frac{d P}{d t}
$$

$$
=-\operatorname{KPVd} \frac{\mathrm{dI}_{\mathrm{d}}}{\mathrm{dt}} \cdot(16)
$$

Considering $\mathrm{Vd}$ and Pref as constant if $\mathrm{SP}(\mathrm{eP}, \mathrm{t})>0$, then it must be $\dot{S P}(e P, t)<0$

from (16) if $\mathrm{KP} \mathrm{Vd}$ is positive thend $\mathrm{I}_{\mathrm{d}} / \mathrm{dt}>0$, meaning that $\mathrm{P}$ must increase.

from the equivalent model in dq coordinates in (1) if the chosen vector has $\mathrm{V}_{\mathrm{Ld}}>\mathrm{V}_{\mathrm{Rod}}-\omega \mathrm{I}_{\mathrm{q}} \mathrm{L}_{2}+\mathrm{R}_{2} \mathrm{I}_{\mathrm{d}}$ then $\mathrm{dI}_{\mathrm{d}} / \mathrm{dt}>0$ ,the selected vector being suitable to increase the active power. b)from (5) and (13) with reactive power Qref and Vd in steady state

$$
\begin{gathered}
\dot{S Q}(e Q, t)=k Q\left(\frac{d Q r e f}{d t}-\frac{d Q}{d t}\right)=-k Q \frac{d Q}{d t} \\
=K Q V d \frac{d_{1}}{d t} .
\end{gathered}
$$

if SQfeQ,t) $>0$, then $\operatorname{SQ}(e P, t)<0$ which still impliesdQ/dt $>0$, meaning that $\mathrm{Q}$ must increase. from (17) $\mathrm{dI}_{\mathrm{q}} / \mathrm{dt}$ must be negative from the equivalent model in $\mathrm{dq}$ coordinates in (2) to ensure the reaching condition, the chosen vector has $\mathrm{V}_{\mathrm{Lq}}<\mathrm{V}_{\mathrm{Roq}}+\omega \mathrm{I}_{\mathrm{d}} \mathrm{L}_{2}+\mathrm{R}_{2} \mathrm{I}_{\mathrm{q}}$, means that voltage vector has $\mathrm{q}$ component suitable to increase the reactive power.

The criteria to choose the matrix vector should be:

1) $\mathrm{SP}(\mathrm{eP}, \mathrm{t})>0=>\operatorname{SP}(\mathrm{eP}, \mathrm{t})<0 \Rightarrow \mathrm{P}<$ Pref

Then choose a vector suitable to increase $P$.

2) $\mathrm{SP}(\mathrm{eP}, \mathrm{t})<0=>\dot{\mathrm{SP}}(\mathrm{eP}, \mathrm{t})>0=>\mathrm{P}>$ Pref

Then choose a vector suitable to decrease $P$.

3 . If $\mathrm{SP}(\mathrm{eP}, \mathrm{t})=0,(18)$

Then choose a vector which does not significantly change the active power.

The same can be applied to reactive power error.

c) control of matrix converter input reactive power matrix converter UPFC can control the reactive power at its input .voltage source input filter (Fig 3) has a relative degree of two then suitable sliding surface SQi(eQi,t) will be:

SQi $(e Q i, t)=($ Qiref- Qi $)+K_{Q i} \frac{d}{d t}\left(Q_{\text {iref }}-Q_{i}\right)$

KQi is chosen to obtain a suitable switching frequency.

By considering (6) in above equation iq, matrix input current must have enough amplitude to fulfil stability.

1.SQi $(e Q i, t)>0 \Rightarrow \operatorname{SQi}(e Q i, t)<0$, then select vector with current iq $<0$ to increase Qi

2.SQi $(e Q i, t)<0=>\operatorname{SQi}(e Q i, t)>0$, then

Select vector with current iq $>0$ to decrease Qi .

The sign of matrix converter reactive power Qiis determined by knowing the location of the input voltages and the location 
of the output currents in Fig:5(input current depends on output currents from TABLE I).

To ease the vector selection Sliding surfaces $\mathrm{SP}(\mathrm{eP}, \mathrm{t})$ and $\mathrm{SQ}(\mathrm{eQ}, \mathrm{t})$ should be transformed to $\alpha \beta$ coordinates $\mathrm{S} \alpha(\mathrm{eP}, \mathrm{t})$ and $\mathrm{S} \beta(\mathrm{eQ}, \mathrm{t})$.

If the control errors eP and $\mathrm{eQ}$ are quantised using two hysterises comparators each with three levels $(-1,0,1)$ nine output voltage vector error combinations are obtained if a two level comparator is used to control the shunt reactive power ,18 error combinations will be defined ,enabling the selection of 18 vectors.

The sliding surfaces for input reactive power is quantised in two levels $(-1$ and +1$)$ using one hysteresis comparator.

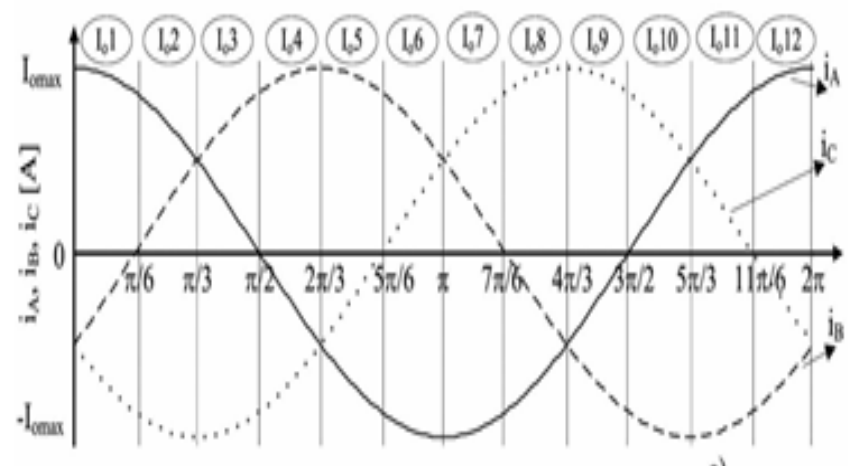

a)

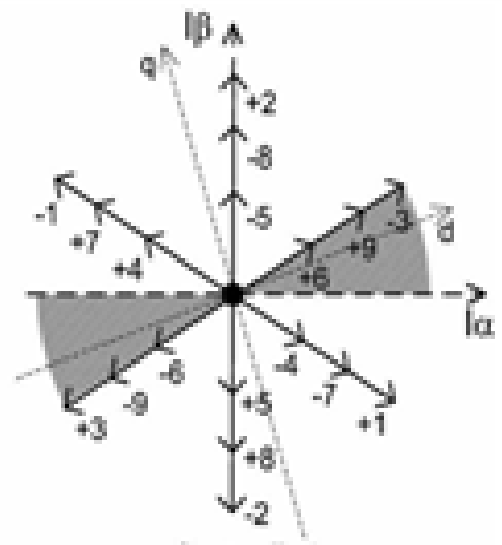

Satior I,

b)

Fig.5: (a)Output currents and their corresponding sector.(b)Input current state-space vectors, when output currents a relocated at sector $\mathrm{I}_{0} 1$. The $\mathrm{dq}$ axis is represented, considering that the input voltages are located in zone $\mathrm{V}_{\mathrm{i}} 1$.

As an example consider the case of $\mathrm{C} \alpha=\mathrm{S} \alpha(\mathrm{eP}, \mathrm{t})>0$ and $\mathrm{C} \beta=$ $\mathrm{S} \beta(\mathrm{eQ}, \mathrm{t})<0$. Then, $\mathrm{dP} / \mathrm{dt}>0$ and $\mathrm{dQ} / \mathrm{dt}<0$ imply that $\mathrm{dI}_{\alpha} / \mathrm{dt}>0$ and $\mathrm{dI}_{\beta} / \mathrm{dt}>0$. To choose the adequate output voltage vector it is necessary to know the input voltage location suppose, it is in Vi1 [fig 4(b)] then vector to be applied should be +9 or -7 Using the same reasoning for the remaining eight active and reactive power error combinations and generalize it for all other input voltage sectors, TABLE II is obtained.

The final choice between +9 and -7 depends on the matrix reactive power controller CQi ,However, at sector Io1, if iq has a suitable amplitude, vector +9 leads to $\operatorname{SQi}(e Q, t)>0$ while vector -7 originates $\operatorname{SQi}(e Q, t)<0$.So, vector +9 is chosen if input reactive power sliding surface $\mathrm{SQi}(\mathrm{eQi}, \mathrm{t})$ is quantised as CQi= -1 , while

vector -7 is chosen when SQi(eQi,t) is quantised as $\mathrm{CQi}=1$ Using same for remaining eight combination at sector Vi1 and applying it for the other output current sectors, TABLE III is obtained shown below: 
TABLE-II STATE SPACE VECTORS SELECTION FOR DIFFERENT ERROR COMBINATIONS

\begin{tabular}{|c|c|c|c|c|c|c|c|}
\hline \multirow{2}{*}{$C_{\alpha}$} & \multirow{2}{*}{$\mathbf{C}_{\beta}$} & \multicolumn{6}{|c|}{ Sector } \\
\hline & & $V_{i} 12 ; 1$ & $V_{1} 2 ; 3$ & $V_{i} 4 ; 5$ & $V_{i} 6 ; 7$ & $V_{i} 8 ; 9$ & $V_{i} 10 ; 11$ \\
\hline-1 & +1 & $-9 ;+7$ & $-9 ;+8$ & $+8 ;-7$ & $-7 ;+9$ & $+9 ;-8$ & $-8 ;+7$ \\
\hline-1 & 0 & $+3 ;-1$ & $+3 ;-2$ & $-2 ;+1$ & $+1 ;-3$ & $-3 ;+2$ & $+2 ;-1$ \\
\hline-1 & -1 & $-6 ;+4$ & $-6 ;+5$ & $+5 ;-4$ & $-4 ;+6$ & $+6 ;-5$ & $-5 ;+4$ \\
\hline 0 & +1 & $-9 ;+7 ;+6 ;-4$ & $-9 ;+8 ;+6 ;-5$ & $+8 ;-7 ;-5 ;+4$ & $-7 ;+9 ;+4 ;-6$ & $+9 ;-8 ;-6 ;+5$ & $-8 ;+7 ;+5 ;-4$ \\
\hline 0 & $\mathbf{0}$ & $\begin{array}{c}\mathrm{Za} ; \mathrm{Zb} ; \mathrm{Zc} ; \\
-8 ;+2 ;-5 ;+8 ;-2 ;+5\end{array}$ & $\begin{array}{c}\mathrm{Za} ; \mathrm{Zb} ; \mathrm{Zc} ; \\
-7 ;+1 ;-4 ;+7 ;-1 ;+4\end{array}$ & $\begin{array}{c}\mathrm{Za} ; \mathrm{Zb} ; \mathrm{Zc} ; \\
+9 ;-3 ;+6 ;-9 ;+3 ;-6\end{array}$ & $\begin{array}{c}\mathrm{Za} ; \mathrm{Zb} ; \mathrm{Zc} ; \\
-8 ;+2 ;-5 ;+8 ;-2 ;+5\end{array}$ & $\begin{array}{c}\mathrm{Za} ; \mathrm{Zb} ; \mathrm{Zc} ; \\
-7 ;+1 ;-4 ;+7 ;-1 ;+4\end{array}$ & $\begin{array}{c}\mathrm{Za} ; \mathrm{Zb} ; \mathrm{Zc} ; \\
-9 ;+3 ;-6 ;+9 ;-3 ;+6\end{array}$ \\
\hline 0 & -1 & $-6 ;+4 ;+9 ;-7$ & $+5 ;-6 ;-8 ;+9$ & $+5 ;-4 ;-8 ;+7$ & $-4 ;+6 ;+7 ;-9$ & $+6 ;-5 ;-9 ;+8$ & $-5 ;+4 ;+8 ;-7$ \\
\hline+1 & +1 & $+6 ;-4$ & $+6 ;-5$ & $-5 ;+4$ & $+4 ;-6$ & $-6 ;+5$ & $+5 ;-4$ \\
\hline+1 & 0 & $-3 ;+1$ & $+2 ;-3$ & $-1 ;+2$ & $+3 ;-1$ & $-2 ;+3$ & $+1 ;-2$ \\
\hline+1 & -1 & $+9 ;-7$ & $+9 ;-8$ & $+7 ;-8$ & $+7 ;-9$ & $-9 ;+8$ & $+8 ;-7$ \\
\hline
\end{tabular}

TABLE III STATE SPACE VECTROS SELECTION,FOR INPUT VOLTAGES LOCATED AT DIFFERENT VOLTAGE SECTORS

\begin{tabular}{|c|c|c|c|c|c|c|}
\hline \multirow{3}{*}{$\begin{array}{l}\mathrm{C} \alpha \mathrm{C} \beta \\
\mathrm{Vi1}\end{array}$} & Io12,Io1 & Io2;Io3 & I04;Io5 & Io6;Io7 & Io8;Io9 & Io10;Io11 \\
\hline & CQi & CQi & CQi & CQi & CQi & CQi \\
\hline & $\begin{array}{lll}+1 & -1\end{array}$ & $+1-1$ & $\begin{array}{lll}+1 & -1\end{array}$ & $\begin{array}{lll}+1 & -1\end{array}$ & $+1 \quad-1$ & $\begin{array}{lll}+1 & -1\end{array}$ \\
\hline$-1+1$ & $-9+7$ & $\begin{array}{ll}-9 & +7\end{array}$ & $-9+7$ & $\begin{array}{ll}+7 & -9\end{array}$ & $+7-9$ & $+7-9$ \\
\hline \begin{tabular}{ll|}
-1 & 0
\end{tabular} & $\begin{array}{ll}+3 & -1 \\
\end{array}$ & $+3-1$ & $-1+3$ & $\begin{array}{|ll|}-1 & +3\end{array}$ & $-1+3$ & $\begin{array}{ll}+3 & -1 \\
\end{array}$ \\
\hline $\begin{array}{lll}-1 & -1\end{array}$ & $-6+4$ & $+4-6$ & $+4-6$ & $+4-6$ & $-6+4$ & $-6+4$ \\
\hline $0+1$ & $-9+7$ & $\begin{array}{ll}-9 & +7\end{array}$ & $\begin{array}{ll}-9 & +7\end{array}$ & $\begin{array}{ll}+7 & -9\end{array}$ & $\begin{array}{ll}+7-9 \\
\end{array}$ & $+7-9$ \\
\hline $\begin{array}{|ll|}0 & 0 \\
\end{array}$ & $-2+2$ & $\begin{array}{ll}+8 & -8\end{array}$ & $-5+5$ & $\begin{array}{ll}+2 & -2\end{array}$ & $-8+8$ & $\begin{array}{ll}+5 & -5\end{array}$ \\
\hline $\begin{array}{|ll|}0 & -1 \\
\end{array}$ & $-7+9$ & $-7+9$ & $-7+9$ & $+9-7$ & $+9-7$ & $+9-7$ \\
\hline$+1+1$ & $-4+6$ & $+6-4$ & $+6-4$ & $\begin{array}{l}+6-4 \\
\end{array}$ & $-4+6$ & $-4+6$ \\
\hline $\begin{array}{ll}+1 & 0\end{array}$ & $\begin{array}{ll}+1 & -3 \\
\end{array}$ & $\begin{array}{lll}+1 & -3\end{array}$ & $-3+1$ & $\begin{array}{ll}-3+1 \\
\end{array}$ & $-3+1$ & $\begin{array}{lll}+1 & -3\end{array}$ \\
\hline $\begin{array}{ll}+1 & -1\end{array}$ & $-7+9$ & $-7+9$ & $-7+9$ & $\begin{array}{l}+9-7 \\
\end{array}$ & $+9-7$ & $+9-7$ \\
\hline
\end{tabular}

\begin{tabular}{|c|c|c|c|c|c|c|}
\hline \multirow{3}{*}{$\begin{array}{l}\mathrm{C} \alpha \mathrm{C} \beta \\
\mathrm{Vi} 2\end{array}$} & Io12,Io1 & Io2;Io3 & I04;Io5 & Io6;Io7 & Io8;Io9 & Io10;Io11 \\
\hline & CQi & CQi & CQi & CQi & \multirow{2}{*}{$\begin{array}{l}\text { CQi } \\
+1-1\end{array}$} & CQi \\
\hline & $+1-1$ & $+1-1$ & $+1 \quad-1$ & $+1-1$ & & $+1-1$ \\
\hline$-1+1$ & $-9+8$ & $\begin{array}{ll}-9 & +8\end{array}$ & $-9+8$ & $+8-9$ & $+8-9$ & $+8-9$ \\
\hline $\begin{array}{ll}-1 & 0\end{array}$ & $+3-2$ & $+3-2$ & $-2+3$ & $-2+3$ & $-2+3$ & $+3-2$ \\
\hline $\begin{array}{ll}-1 & -1\end{array}$ & $-6+5$ & $+5-6$ & $+5-6$ & $+5-6$ & $-6+5$ & $-6+5$ \\
\hline $0+1$ & $-9+8$ & $-9+8$ & $\begin{array}{ll}-9 & +8\end{array}$ & $+8-9$ & $+8-9$ & $+8-9$ \\
\hline $\begin{array}{ll}0 & 0\end{array}$ & $-1+1$ & $+7-7$ & $\begin{array}{ll}-4 & +4\end{array}$ & $\begin{array}{ll}+1 & -1\end{array}$ & $-7+7$ & $+4-4$ \\
\hline $\begin{array}{ll}0 & -1 \\
\end{array}$ & $+9-8$ & $+9-8$ & $+9-8$ & $-8+9$ & $-8+9$ & $-8+9$ \\
\hline$+1+1$ & $-5+6$ & $+6-5$ & $+6-5$ & $+6-5$ & $-5+6$ & $-5+6$ \\
\hline $\begin{array}{ll}+1 & 0\end{array}$ & $-3+2$ & $-3+2$ & $+2 \quad-3$ & $+2 \quad-3$ & $-3-3$ & $-3+2$ \\
\hline $\begin{array}{ll}+1 & -1\end{array}$ & $-8+9$ & $-8+9$ & $-8+9$ & $+9-8$ & $+9-8$ & $+9-8$ \\
\hline
\end{tabular}




\begin{tabular}{|c|c|c|c|c|c|c|}
\hline \multirow{3}{*}{$\begin{array}{l}\mathrm{C} \alpha \mathrm{C} \beta \\
\mathrm{Vi3}\end{array}$} & Io12,Io1 & Io2;Io3 & I04;Io5 & Io6;Io7 & Io8;Io9 & $\begin{array}{l}\text { Io10; } \\
\text { Io11 }\end{array}$ \\
\hline & CQi & CQi & CQi & CQi & CQi & CQi \\
\hline & $+1 \quad-1$ & $+1 \quad-1$ & $+1-1$ & $+1-1$ & $+1-1$ & $+1-1$ \\
\hline$-1+1$ & $\begin{array}{ll}+8 & -7\end{array}$ & $+8 \quad-7$ & $+8-7$ & $\begin{array}{ll}-7 & +8\end{array}$ & $\begin{array}{ll}-7 & +8\end{array}$ & $\begin{array}{r}-7 \\
+8\end{array}$ \\
\hline $\begin{array}{ll}-1 & 0\end{array}$ & $-2+1$ & $-2+1$ & $+1-2$ & $+1-2$ & $+1-2$ & $\begin{array}{r}-2 \\
+1\end{array}$ \\
\hline$-1 \quad-1$ & $\begin{array}{ll}+5 & -4\end{array}$ & $-4+5$ & $-4 \quad+5$ & $-4+5$ & $\begin{array}{ll}+5 & -4\end{array}$ & $\begin{array}{ll}+5 & - \\
4\end{array}$ \\
\hline $0+1$ & $+8 \quad-7$ & $+8 \quad-7$ & $+8-7$ & $-7+8$ & $-7+8$ & $-7+8$ \\
\hline $\begin{array}{ll}0 & 0\end{array}$ & $+3-3$ & $-9+9$ & $+6-6$ & $-3+3$ & $\begin{array}{ll}+9 & -9\end{array}$ & $\begin{array}{r}-6 \\
+6\end{array}$ \\
\hline $\begin{array}{ll}0 & -1\end{array}$ & $+7-8$ & $+7-8$ & $+7-8$ & $-8+7$ & $\begin{array}{ll}-8 & +7\end{array}$ & $\begin{array}{r}-8 \\
+7\end{array}$ \\
\hline$+1+1$ & $+4-5$ & $-5+4$ & $-5+4$ & $-5+4$ & $\begin{array}{ll}+4 & -5\end{array}$ & $\begin{array}{ll}+4 & - \\
5 & \end{array}$ \\
\hline+10 & $+2-1$ & $+2-1$ & $\begin{array}{ll}-1 & +2\end{array}$ & $-1+2$ & $-1+2$ & $\begin{array}{ll}+2 & - \\
1 & \end{array}$ \\
\hline $\begin{array}{ll}+1 & -1\end{array}$ & $-8+7$ & $-8+7$ & $-8+7$ & $\begin{array}{ll}+7 & -8\end{array}$ & $\begin{array}{ll}+7 & -8\end{array}$ & $\begin{array}{ll}+7 & - \\
8 & \end{array}$ \\
\hline
\end{tabular}

\begin{tabular}{|c|c|c|c|c|c|c|}
\hline $\begin{array}{l}\mathrm{C} \alpha \mathrm{C} \beta \\
\mathrm{Vi} 4\end{array}$ & Io12,Io1 & Io2;Io3 & I04;Io5 & Io6;Io7 & Io8;Io9 & Io10;Io11 \\
\hline & CQi & CQi & CQi & CQi & CQi & CQi \\
\hline & $+1-1$ & $+1-1$ & $+1-1$ & $+1-1$ & $+1-1$ & $+1-1$ \\
\hline$-1+1$ & $\begin{array}{ll}-7 & 9\end{array}$ & $\begin{array}{ll}-7 & 9\end{array}$ & $-7+9$ & $+9-7$ & $9-7$ & $9-7$ \\
\hline $\begin{array}{ll}-1 & 0\end{array}$ & $+1-3$ & $\begin{array}{ll}+1 & -3\end{array}$ & $-3+1$ & $-3+1$ & $-3+1$ & $+1 \quad-3$ \\
\hline$-1 \quad-1$ & $-4+6$ & $\begin{array}{ll}+6 & -4\end{array}$ & $+6-4$ & $+6-4$ & $-4+6$ & $-4+6$ \\
\hline $\begin{array}{ll}0 & 0\end{array}$ & $-2+2$ & $+8-8$ & $-5+5$ & $-2+2$ & $-8+8$ & $+5 \quad-5$ \\
\hline $\begin{array}{ll}0 & -1\end{array}$ & $\begin{array}{ll}-9 & +7\end{array}$ & $\begin{array}{ll}-9 & +7\end{array}$ & $-9+7$ & $\begin{array}{ll}+7 & -9\end{array}$ & $\begin{array}{ll}+7 & -9\end{array}$ & $\begin{array}{ll}+7 & -9\end{array}$ \\
\hline$+1+1$ & $-6+4$ & $+4-6$ & $+4-6$ & $+4-6$ & $-6+4$ & $-6+4$ \\
\hline+10 & $-1+3$ & $\begin{array}{ll}-1 & +3\end{array}$ & $\begin{array}{ll}+3 & -1\end{array}$ & $\begin{array}{ll}+3 & -1\end{array}$ & $\begin{array}{ll}+3 & -1\end{array}$ & $-1+3$ \\
\hline$+1-1$ & $-9+7$ & $-9+7$ & $\begin{array}{ll}-9 & +7\end{array}$ & $+7-9$ & $+7-9$ & $+7-9$ \\
\hline
\end{tabular}




\begin{tabular}{|c|c|c|c|c|c|c|}
\hline $\mathrm{C} \alpha \mathrm{C} \beta$ & Io12,Io1 & Io2;Io3 & I04;Io5 & Io6;Io7 & Io8;Io9 & Io10;Io11 \\
\hline & CQi & CQi & CQi & CQi & CQi & CQi \\
\hline & $+1-1$ & $+1-1$ & $+1-1$ & $+1-1$ & $+1-1$ & $+1-1$ \\
\hline$-1+1$ & $+9-8$ & $+9-8$ & $+9-8$ & $-8+9$ & $-8+9$ & $-8+9$ \\
\hline $\begin{array}{ll}-1 & 0\end{array}$ & $-3+2$ & $-3+2$ & $\begin{array}{ll}+2 & -3\end{array}$ & $\begin{array}{ll}+2 & -3\end{array}$ & $+2 \quad-3$ & $-3+2$ \\
\hline$-1-1$ & $+6-5$ & $-5+6$ & $-5+6$ & $-5+6$ & $+6-5$ & $+6-5$ \\
\hline $0+1$ & $+9-8$ & $+9-8$ & $+9-8$ & $-8+9$ & $-8+9$ & $-8+9$ \\
\hline $\begin{array}{ll}0 & 0\end{array}$ & $-1+1$ & $+7-7$ & $-4+4$ & $\begin{array}{ll}+1 & -1\end{array}$ & $-7+7$ & $+4-4$ \\
\hline $0-1$ & $+8-9$ & $+8-9$ & $+8-9$ & $-9+8$ & $-9+8$ & $-9+8$ \\
\hline$+1+1$ & $+5-6$ & $-6+5$ & $-6+5$ & $-6+5$ & $+5-6$ & $+5-6$ \\
\hline+10 & $+3-2$ & $+3-2$ & $-2+3$ & $-2+3$ & $-2+3$ & $+3-2$ \\
\hline$+1-1$ & $+8-9$ & $+8-9$ & $+8-9$ & $-9+8$ & $-9+8$ & $-9+8$ \\
\hline
\end{tabular}

\begin{tabular}{|c|c|c|c|c|c|c|}
\hline $\mathrm{C} \alpha \mathrm{C} \beta$ & Io12,Io1 & Io2;Io3 & I04;Io5 & Io6;Io7 & Io8;Io9 & Io10;Io11 \\
\hline \multirow[t]{2}{*}{ Vi6 } & CQi & CQi & CQi & CQi & \multirow{2}{*}{$\begin{array}{l}\text { CQi } \\
+1 \quad-1\end{array}$} & CQi \\
\hline & $+1-1$ & $+1-1$ & $+1 \quad-1$ & $\begin{array}{ll}+1 & -1\end{array}$ & & $+1-1$ \\
\hline$-1+1$ & $-8+7$ & $-8+7$ & $-8+7$ & $+7-8$ & $+7-8$ & $+7-8$ \\
\hline $\begin{array}{ll}-1 & 0\end{array}$ & $+2-1$ & $+2-1$ & $-1+2$ & $-1+2$ & $-1+2$ & $+2-1$ \\
\hline$-1 \quad-1$ & $-5+4$ & $+4-5$ & $+4-5$ & $+4-5$ & $-5+4$ & $-5+4$ \\
\hline $0+1$ & $-8+7$ & $-8+7$ & $-8+7$ & $+7-8$ & $+7-8$ & $+7-8$ \\
\hline $\begin{array}{ll}0 & 0\end{array}$ & $+3-3$ & $-9+9$ & $+6-6$ & $-3+3$ & $\begin{array}{ll}+9 & -9\end{array}$ & $-6+6$ \\
\hline $0-1$ & $\begin{array}{ll}-7 & +8\end{array}$ & $\begin{array}{ll}-7+8 \\
\end{array}$ & $\begin{array}{ll}-7 & +8\end{array}$ & $+8 \quad-7$ & $+8 \quad-7$ & $+8 \quad-7$ \\
\hline$+1+1$ & $-4+5$ & $+5-4$ & $+5-4$ & $+5-4$ & $-4+5$ & $-4+5$ \\
\hline+10 & $-2+1$ & $-2+1$ & $+1-2$ & $+1-2$ & $+1-2$ & $-2+1$ \\
\hline$+1 \quad-1$ & $-7+8$ & $-7+8$ & $-7+8$ & $+8-7$ & $+8-7$ & $+8-7$ \\
\hline
\end{tabular}




\section{IMPLEMENTATION OF THE MCAS UPFC:}

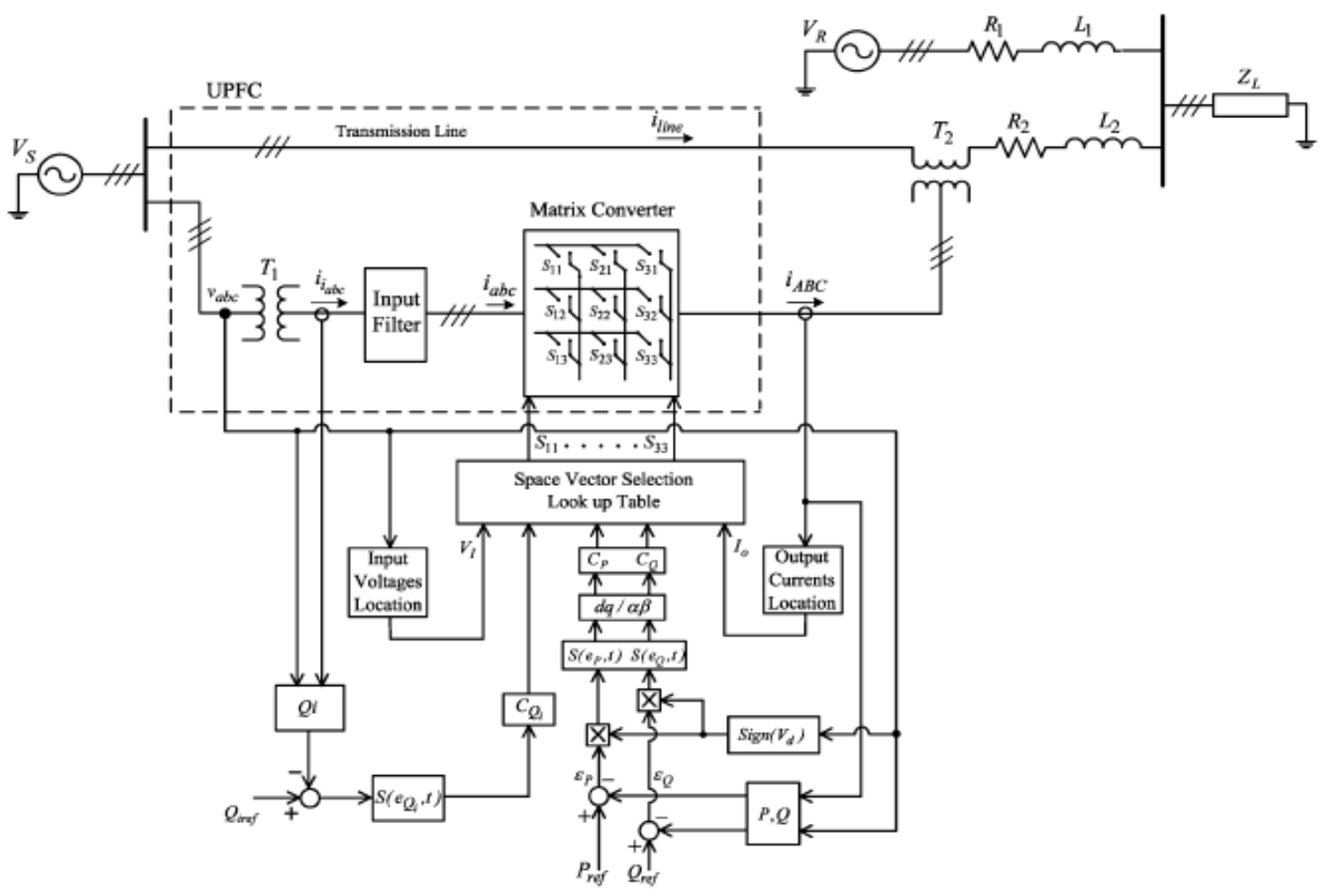

Fig 6 Control scheme of DPC of the three phase matrix converter operating as the UPFC

\section{SIMULATION AND EXPERIMENTAL RESULT}

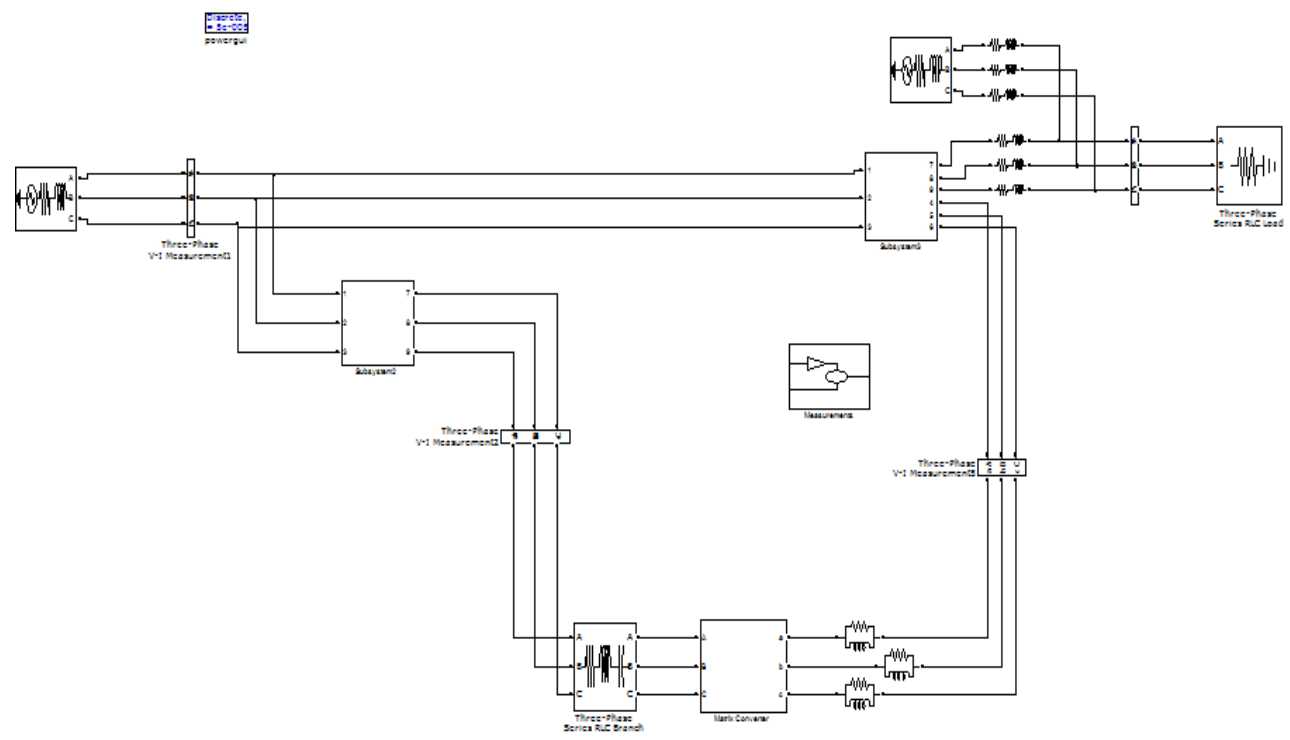

Transmission network with matrix converter - UPFC 


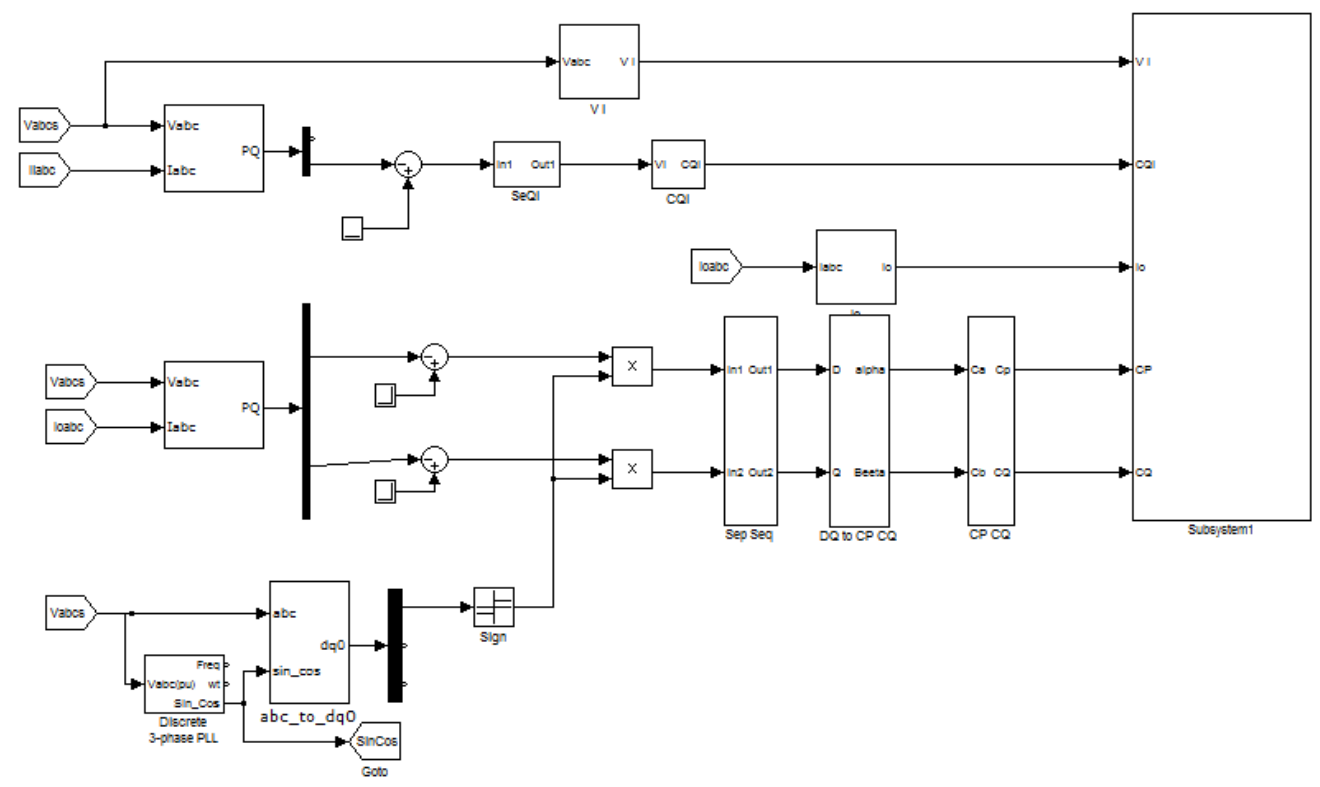

Control scheme of matrix converter as UPFC using space vector selection table

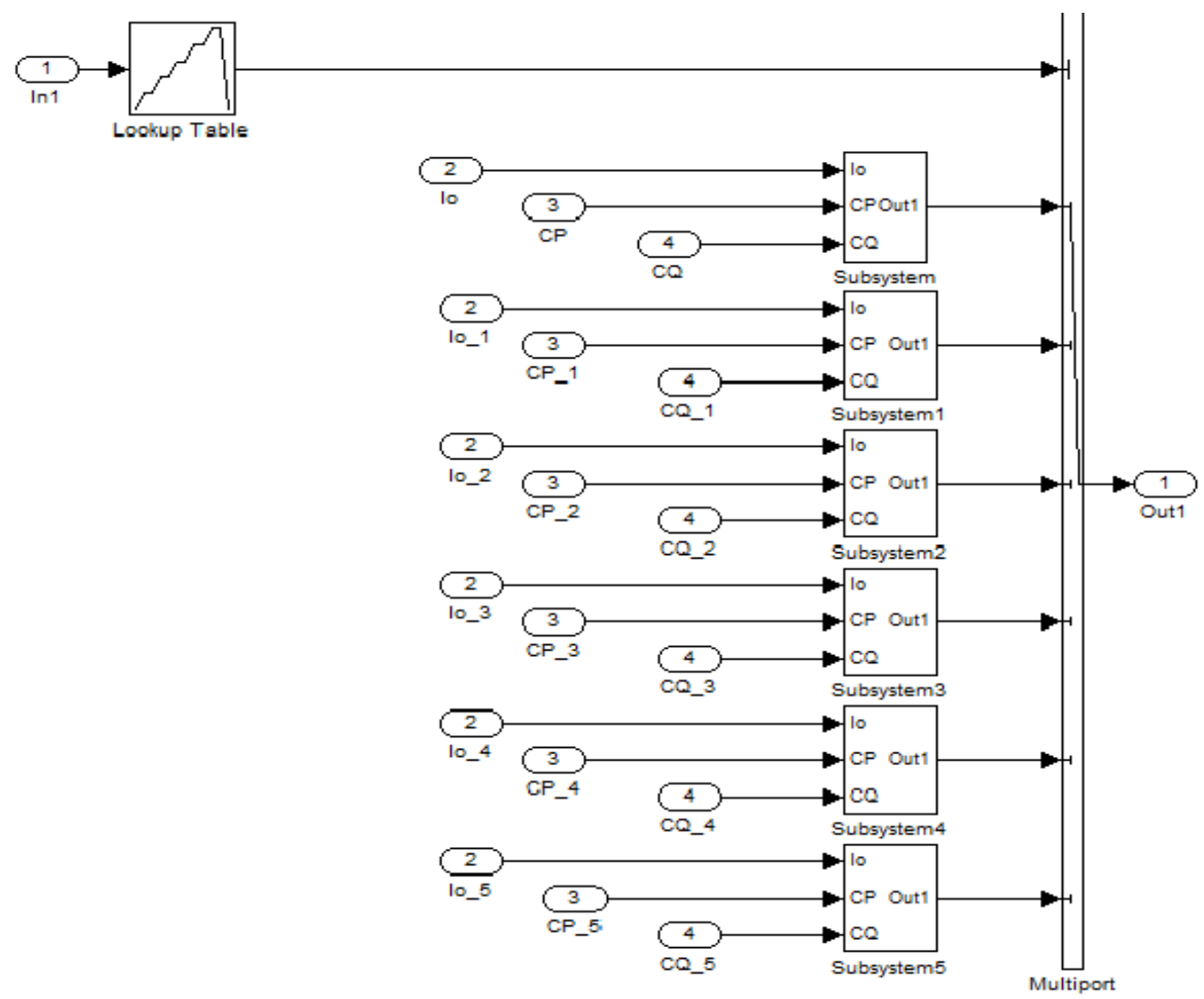

State space vector selection 
Fig.7(a)and(b)shows, respectively, the simulation results of the active and reactive power step response $(\Delta$ Pref $=+0.4$ p.u.and $\Delta$ Qref $=+0.2$ p.u.) and shunt reactive power, considering initial reference values: Pref=0.4p.u.,Qref $=0.2$ p.u., and Qref=-0.07p.u.Bothresultsclearlyshowthatthereis no cross-coupling between active and reactive power.
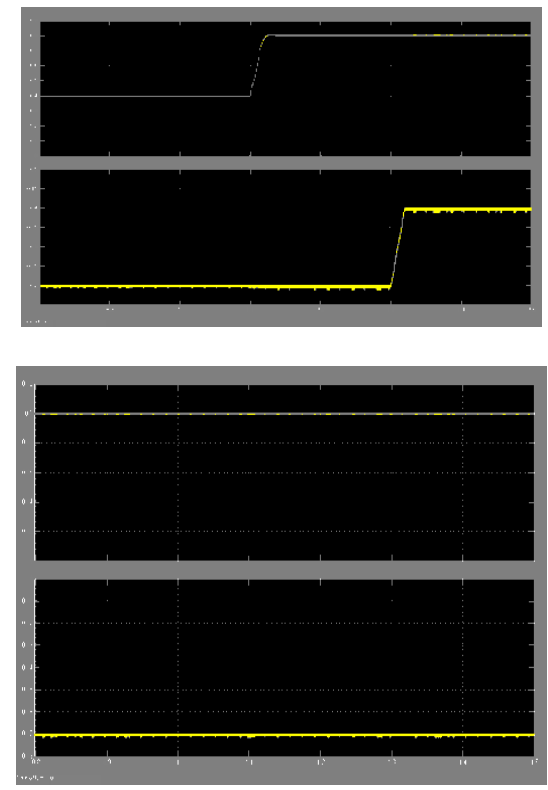

Fig7(a),(b): Active and reactive series power response and reactive shunt power, for $\mathrm{P}$ and $\mathrm{Q}$ steps

TheresultsofFig.8(a)and(b)showlineandinputmatrixconverterc urrentsinsteady state, for Pref $=0.4$ p.u., Qref=0.2p.u.,andQref=-0.07p.u.Currentsare almost sinusoidal with small ripple content.

DPCsimulationresultspresentedinFig.9(a)and(b), showingthecla imedDPCfasterdynamicresponsetostepactiveandreactivepower referencechange.

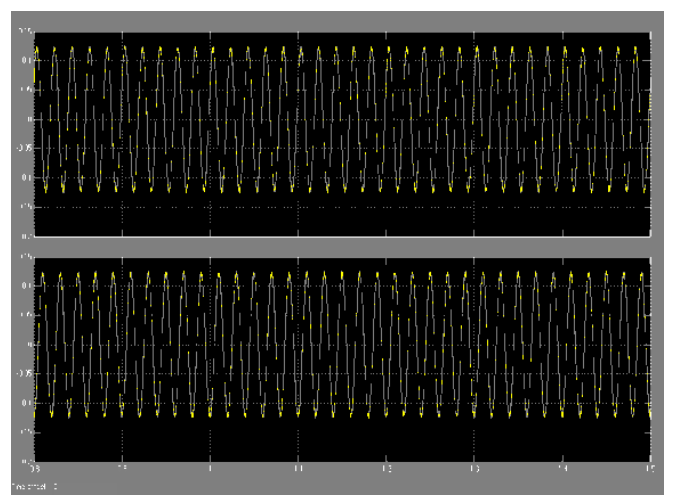

Fig.8(a),(b): Line currents (iA,iB) and input matrix converter currents(ib, ic) pu. Simulation results
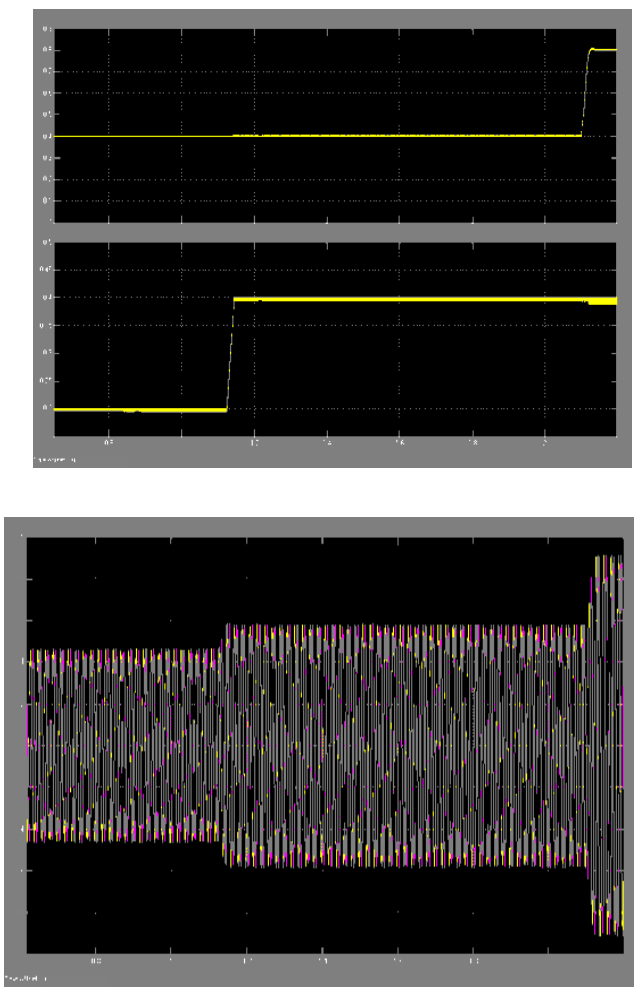

Fig9(a),(b): Active and reactive power response and line currents for a P and Q step change Direct power controller simulations

DPC controller ability to operate at lower switching frequencies, the DPC gains were lowered and the input filter parameters were changed accordingly $(\mathrm{r}=25 \Omega, \mathrm{l}=5.9 \mathrm{~m}$ $\mathrm{HC}=12.6 \mu \mathrm{F}$ ) to lower the switching frequencytonearly $1.4 \mathrm{kHz}$. The results (Fig.10) also show fast response without cross coupling between active and reactive power. This confirms the DPC-MC robustness to input filter parameter variation, the ability to operate at low switching frequencies, and insensitivity to Switching nonlinearity.

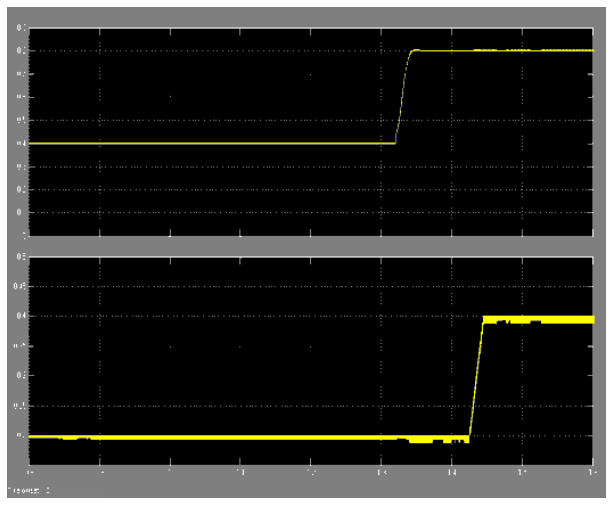




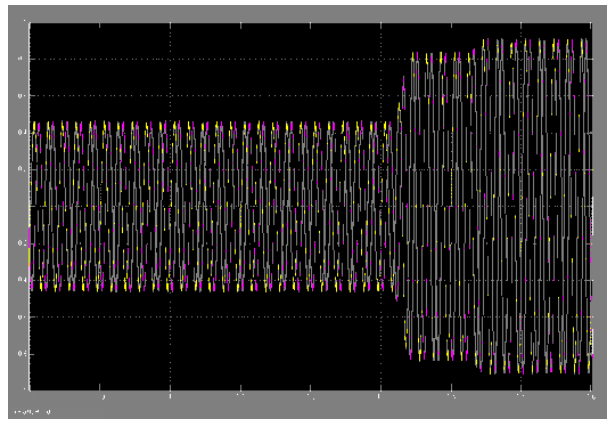

Fig10 (a)(b): Active and reactive power response and line currents for a $\mathrm{P}$ and $\mathrm{Q}$

\section{CONCLUSIONS}

This paper derived advanced non linear direct power controllers ,for matrix converters connected to power transmission lines as UPFCs. Presented simulation \& experimental results show that active and reactive flow will be advantageously controlled by using the proposed DPC. Results show no steady-state errors, no cross coupling, insensitivity to non modelled dynamics and fast response times, thus confirming the expected performance of the presented nonlinear DPC methodology. Despite showing a suitable dynamic response, the PI performance is inferior when compared to DPC. Furthermore, the PI controllers and modulator take longer times to compute. Obtained results show that DPC is a strong non linear control candidate for line active and reactive power flow.

\section{REFERENCES}

[1]. N. Hingorani and L. Gyugyi, Understanding FACTSConcepts and Technology of Flexible AC Transmission Systems. Piscataway, NJ:IEEE Press/Wiley, 2000.

[2] L. Gyugyi, "Unified power flow control concept for flexible AC transmission systems," Proc. Inst. Elect. Eng. C, vol. 139, no. 4, Jul. 1992.

[3] L. Gyugyi, C. Schauder, S. Williams, T. Rietman, D. Torgerson, and A. Edris, "The unified power flow controller: A new approach to power transmission control," IEEE Trans. Power Del., vol. 10, no. 2, pp. 1085-1097, Apr. 1995.

[4] C. Schauder, L. Gyugyi, M. Lund, D. Hamai, T. Rietman, D. Torgerson, and A. Edris, "Operation of the unified power flow controller (UPFC) under practical constraints," IEEE Trans. Power Del., vol. 13, no. 2, pp. 630-639, Apr. 1998.

[5] T. Ma, "P-Q decoupled control schemes using fuzzy neural networks for the unified power flow controller," in Electr. Power Energy Syst.. New York: Elsevier, Dec. 2007, vol. 29, pp. 748-748.

[6] L. Liu, P. Zhu, Y. Kang, and J. Chen, "Power-flow control performance analysis of a unified power-flow controller in a novel control scheme," IEEE Trans. Power Del., vol. 22, no. 3, pp. 1613-1619, Jul. 2007.

[7] F. Gao and M. Iravani, "Dynamic model of a space vector modulated matrix converter," IEEE Trans. Power Del., vol.
22, no. 3, pp. 1696-1750, Jul. 2007.

[8] B. Geethalakshmi and P. Dananjayan, "Investigation of performance of UPFC without DC link capacitor," in Elect. Power Energy Res.. New York: Elsevier, 2008, pp. 284-294, 736-746.

[9] X. Jiang, X. Fang, J. Chow, A. Edris, E. Uzunovic, M. Parisi, and L. Hopkins, "A novel approach for modeling voltage-sourced converter based FACTS controllers," IEEE Trans. Power Del., vol. 23, no. 4, pp. 2591-2598, Oct. 2008.

[10] R. Strzelecki, A. Noculak, H. Tunia, and K. Sozanski, "UPFC with matrix converter," presented at the EPE Conf., Graz, Austria, Sep. 2001.

[11] J. Monteiro, J. Silva, S. Pinto, and J. Palma, "Unified power flow controllers without DC bus: Designing controllers for the matrix converter solution," presented at the Int. Conf. Electrical Engineering, Coimbra, Portugal, 2005.

[12] A. Dasgupta, P. Tripathy, and P. Sensarma, "Matrix converter as UPFC for transmission line compensation," in Proc. 7th Int. Conf. Power Electronics, Exco, Daegu, Korea, Oct. 2007, pp. 1050-1055.

[13] P. Wheeler, J. Rodriguez, J. Clare, L. Empringham, and A. Weinstein, "Matrix converters: A technology review," IEEE Trans. Ind. Electron., vol. 49, no. 2, pp. 276-288, Apr. 2002.

[14] S. Pinto, "Conversoresmatriciaistrifásicos: generalização do commando vectorialdirecto," Ph.D. dissertation, Instituto Superior TécnicoUniversidadeTécnica de Lisboa, Lisbon, Portugal, Jul. 2003.

[15] T. Podlesak, D. Katsis, P. Wheeler, J. Clare, L. Empringham, and M. Bland, "A 150-kVAvector-controlled matrix converter induction motor drive," IEEE Trans. Ind. Appl., vol. 41, no. 3, pp. 841-847, May/Jun. 2005.

[16] R. Cárdenas, R. Pena, P. Wheeler, J. Clare, and G. Asher, "Control of the reactive power supplied by WECS based on an induction generator fed by a matrix converter," IEEE Trans. Ind. Electron., vol. 56, no. 2, pp. 429-438, Feb. 2009.

[17] A. Alesina and M. G. B. Venturini, "Solid-state power conversion: A Fourier analysis approach to generalized transformer synthesis," IEEETrans. Circuits Syst., vol. CAS28, no. 4, pp. 319-330, Apr. 1981.

[18] P. J. Wheeler, J. Clare, and L. Empringham, "Enhancement of matrix converter output waveform quality using minimized commutation times," IEEE Trans. Ind. Electron., vol. 51, no. 1, pp. 240-244, Feb. 2004.

[19] H. Akagi, Y. Kanazawa, and A. Nabae, "Instantaneous reactive power compensators comprising switching devices without energy storage components," IEEE Trans. Ind. Appl., vol. IA-20, no. 3, pp. 625-630, May/Jun. 1984.

[20] I. Martins, J. Barros, and J. Silva, "Design of crosscoupling free current mode controller for UPFC series converter," in Proc. IEEE Optimizationof Electrical and Electronic Equipment Conf., May 2008, pp. 209-218. 